\title{
Image potential states at transition metal oxide surfaces: A time-resolved two-photon photoemission study on ultrathin $\mathrm{NiO}$ films
}

\author{
K. Gillmeister, ${ }^{1}$ M. Kiel, ${ }^{1}$ and W. Widdra ${ }^{1,2}$ \\ ${ }^{1}$ Institut für Physik, Martin-Luther-Universität Halle-Wittenberg, Halle, Germany \\ ${ }^{2}$ Max-Planck-Institut für Mikrostrukturphysik, Halle, Germany
}

(Received 3 June 2015; revised manuscript received 15 February 2017; published 16 February 2018)

\begin{abstract}
For well-ordered ultrathin films of $\mathrm{NiO}(001)$ on $\mathrm{Ag}(001)$, a series of unoccupied states below the vacuum level has been found. The states show a nearly free electron dispersion and binding energies which are typical for image potential states. By time-resolved two-photon photoemission (2PPE), the lifetimes of the first three states and their dependence on oxide film thickness are determined. For NiO film thicknesses between 2 and 4 monolayers (ML), the lifetime of the first state is in the range of 28-42 fs and shows an oscillatory behavior with increasing thickness. The values for the second state decrease monotonically from 88 fs for 2 ML to 33 fs for 4 ML. These differences are discussed in terms of coupling of the unoccupied states to the layer-dependent electronic structure of the growing $\mathrm{NiO}$ film.
\end{abstract}

DOI: 10.1103/PhysRevB.97.085424

\section{INTRODUCTION}

Image potential states (IPS) form a special class of surface states, in which electrons are bound in front of a polarizable surface. In response to electrons on the vacuum side, polarization charges are induced at the surface region of the bulk. They result in an attractive image potential that can bind the electrons perpendicularly to the surface. Since the first substantiation of image potential states by 2PPE in 1985 [1], this method is nowadays the tool of choice for such investigations. IPS play a decisive role as model systems to study fundamental decay processes of excited electrons. Extensive research has been conducted on IPS on bare metal surfaces [2-9], metal layers on metals [10-12], rare gas adlayers on different metallic substrates [13-20], and organic thin films on metal surfaces [21-27].

Albeit IPS are exclusive surface states, they can provide information about the electronic structure of the surface to which they couple. IPS can be modified by the (surface projected) bulk band structure $[16,17]$ and will be transformed into resonances by interacting with electronic states at similar energies, as has been also shown for quantum-well states $[12,27]$. Strong interactions might even suppress IPS completely [8].

Theoretically, IPS are well understood and the dynamics of electrons in IPS are described well [28-30]. IPS of bare metal surfaces are often treated in the bulk penetration model. The decay processes of electrons in IPS are determined by their interaction with bulk electrons [12]. Larger lifetimes are found for lower penetration of the IPS wave function into the bulk, and

Published by the American Physical Society under the terms of the Creative Commons Attribution 4.0 International license. Further distribution of this work must maintain attribution to the author(s) and the published article's title, journal citation, and DOI. vice versa. In addition, one has to consider the influence of the long-range Coulomb interaction as it influences the available phase space for decay processes strongly [28,31].

IPS of metal surfaces can be decoupled with the help of insulating adsorbate layers [17]. Thereby, the decoupling is most effective for adlayers that do not introduce any electronic states in the energy region of the IPS. The lifetime of IPS in such systems can be one or two orders of magnitude longer than for clean metal surfaces depending on the morphology and electronic structure of the adlayers [17].

Up to now, only a few 2PPE investigations on metal oxides have been reported, whereas none of these investigations focuses on image potential states and their lifetimes [32-37]. For transition-metal oxides with a static dielectric constant $\varepsilon_{0}$ in the range of $4-12$, one expects again a series of IPS converging to the vacuum level as electrons in these states introduce polarization charges at the oxide surface. However, the exact position and especially the lifetime of the IPS depend on the presence of oxide conduction band states below the vacuum level. Here we present a 2PPE study of IPS for ultrathin epitaxial films of the charge-transfer insulator $\mathrm{NiO}$ grown pseudomorphically on $\mathrm{Ag}(001)$ in the thickness range between 2 and $4 \mathrm{ML}$.

$\mathrm{NiO}$ has a fundamental band gap of about $4 \mathrm{eV}$ starting at $1.5 \mathrm{eV}$ below $E_{F}$ for bulk $\mathrm{NiO}$ and $\mathrm{NiO}$ films on $\mathrm{Ag}(001)$ [38-40] as sketched in Fig. 1(b) and determined by photoemission and inverse photoemission. The $\mathrm{Ag}(001)$ substrate exhibits a band gap around the $\bar{\Gamma}$ point that extends from 2 to $6.8 \mathrm{eV}$ above $E_{F}[41,42]$ as is schematically indicated in Figs. 1(a) and 1(b). For ultrathin $\mathrm{NiO}$ films, however, one expects a modified and thickness-dependent band structure due to electron confinement within the oxide film and due to the vicinity of the metal-oxide interface. With increasing thickness it will finally converge to the $\mathrm{NiO}$ bulk electronic structure $[43,44]$. At the $\mathrm{NiO}$ surface, one expects a series of IPS (labeled states 1 and 2 in Fig. 1, indicated in red) below the vacuum level. In the following, we present data for long-living 

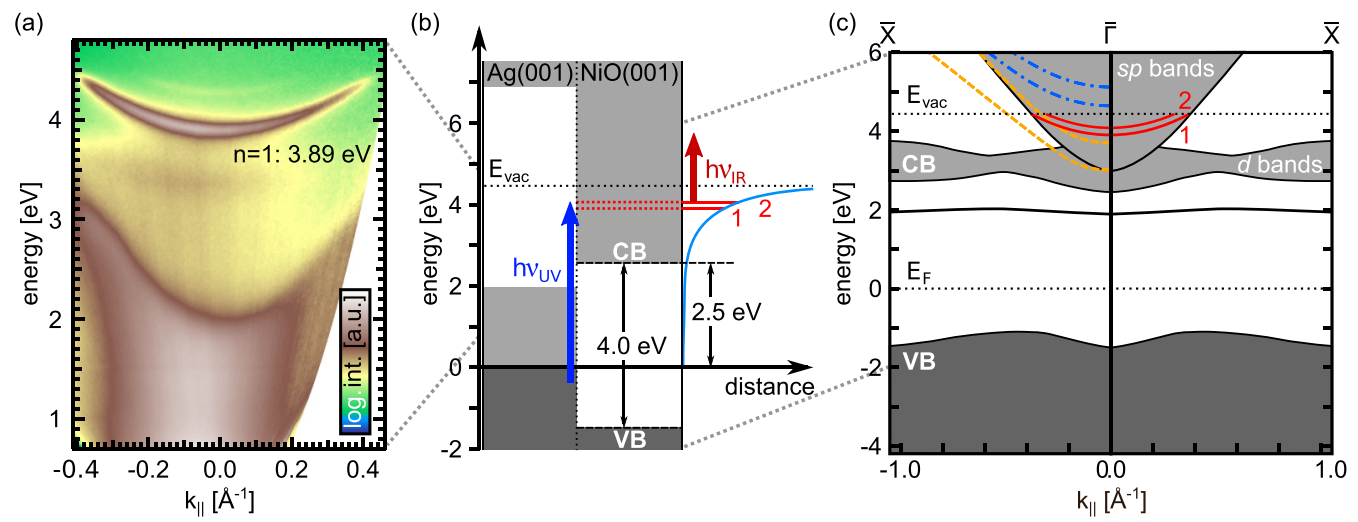

FIG. 1. Band alignment of $\mathrm{NiO}(001) / \mathrm{Ag}(001)$ : (a) 2PPE measurement of the projected band structure of the unoccupied states of $\mathrm{Ag}(001)$ in $\overline{\Gamma X}$ direction using $h v_{\mathrm{UV}}=4.41 \mathrm{eV}$. The $n=1 \mathrm{IPS}$ of $\mathrm{Ag}(001)$ is located at $E_{\mathrm{int}}=3.89 \mathrm{eV}$. (b) Energy level scheme of the system $\mathrm{NiO}(001) / \mathrm{Ag}(001)$ at the $\bar{\Gamma}$ point including the image potential (blue solid line). The unoccupied states 1 and 2 are pumped by $h v_{\mathrm{UV}}$ and probed by $h v_{\mathrm{IR}}$. (c) Schematic surface-projected bulk band structure of $\mathrm{NiO}$ [45] with an energy-shifted conduction band to fit the experimental band gap of $4 \mathrm{eV}$ [38]. In the energy range of interest the theoretical calculations yield $s p$-derived unoccupied states localized at the surface (dashed orange and dashed-dotted blue lines).

unoccupied states near the vacuum level that are assigned to $\mathrm{NiO}$ image potential resonances. Depending on the NiO film thickness in the range between 2 and 4 ML, we find different lifetimes and effective masses of these states that are discussed with respect to coupling to quantum-well and conduction band states. In this way, the present study extends the discussion of IPS to the field of transition metal oxides, where $\mathrm{NiO}$ is the prominent example for a strongly correlated oxide.

\section{EXPERIMENTAL DETAILS}

Sample preparation and all experiments have been performed in an ultrahigh vacuum (UHV) chamber working at a base pressure of $1 \times 10^{-10} \mathrm{mbar}$. The chamber is equipped with an optics for low energy electron diffraction (LEED), an x-ray source for x-ray photoelectron spectroscopy (XPS), as well as a UV source for ultraviolet photoelectron spectroscopy (UPS). The photoemitted electrons are detected by a hemispherical analyzer (Phoibos 150, SPECS, Germany) which contains a CCD detector for simultaneous recording of electron energy and emission angle $\vartheta$. For 2PPE, a broadly tunable femtosecond laser system is used that includes two noncollinear optical parametric amplifiers which are pumped by a $20 \mathrm{~W}$ all-fiber laser (IMPULSE, Clark) as described elsewhere [46,47]. For the experiments described here, UV laser pulses with an energy of 4.17-4.27 eV for optical pumping are combined with infrared (IR) laser pulses (1.68-1.72 eV) for probing with typical pulse lengths of 50-90 fs and 35-45 fs, respectively. In time-resolved experiments, energy-resolved 2PPE spectra are taken for different time delays between pump and probe pulses ( $\Delta t=5 \mathrm{fs}$ ). The cross correlation as well as the time zero between pump and probe pulses are determined simultaneously with the decay of the IPS from the two-dimensional $E(k)$ data. For this purpose, Gaussian fits of the transient 2PPE signals at about $0.5 \mathrm{eV}$ above the low-energy cutoff are used where no long-living unoccupied states are observed [48]. If not stated otherwise, all reported electron energies are referenced to the Fermi energy $E_{F}=0$.
The $\operatorname{Ag}(001)$ crystal was cleaned by several cycles of $\mathrm{Ar}$ ion sputtering and subsequent annealing at $600 \mathrm{~K}$ until no contamination could be detected by XPS. NiO ultrathin films have been grown via molecular beam epitaxy (MBE) as established by Marre and Neddermeyer [49-51]. Thereby, Ni was evaporated from a Ta crucible in an oxygen atmosphere of $2 \times 10^{-6} \mathrm{mbar}$ with a total growth rate of $0.5 \mathrm{ML} / \mathrm{min}$. $\mathrm{NiO}$ films of 2, 3, and $4 \mathrm{ML}$ thickness have been prepared and subsequently annealed at $540 \mathrm{~K}$ to improve long-range order and film perfection. The quality of the films has been monitored by the sharpness of the LEED spots [cf. Fig. 2(a)]. As reported also earlier, we find a layer-by-layer growth for $\mathrm{NiO}$ on $\mathrm{Ag}(001)$ [51-54] for thicknesses starting at $2 \mathrm{ML}$. Hence, after calibrating the evaporator it was possible to prepare well-ordered $\mathrm{NiO}$ layers with an accuracy of $\pm 10 \%$ in thickness.

A combination of LEED, XPS, and work function measurements based on photoemission onset has been used for calibration of the $\mathrm{NiO}$ film thickness. In the monolayer regime, $\mathrm{NiO}$ shows a $(2 \times 1)$ structure $[50,52,53,55,56]$. It is formed by a uniaxially distorted, nearly planar, quasihexagonal $\mathrm{NiO}$ structure [55]. Above $1 \mathrm{ML}$, the $(2 \times 1)$ super cell vanishes and a pseudomorphic rocksaltlike $(1 \times 1)$ structure is formed [53,57]. In Fig. 2(a), diffraction patterns of $1-4 \mathrm{ML} \mathrm{NiO} / \mathrm{Ag}(001)$ are displayed. Intensity profiles along the $\overline{\Gamma X}$ direction give clear evidence that the $(2 \times 1)$ diffraction spots only appear for the $\mathrm{NiO}$ monolayer [Fig. 2(b)]. Figure 2(c) depicts the intensity of the $(2 \times 1)$ LEED spots with increasing coverage (left axis). For the completed monolayer, the $(2 \times 1)$ intensity is maximal. The structural transition from a quasihexagonal monolayer to rocksaltlike $\mathrm{NiO}$ films starting from $2 \mathrm{ML}$ on leads to the disappearance of the $(2 \times 1)$ spots in LEED. Note that thicker $\mathrm{NiO}(001)$ films do show a magnetic $(2 \times 1)$ superstructure that is, however, visible in LEED only at selected electron kinetic energies.

Due to the quasihexagonality, the chemical environment as well as the resulting surface dipole of the $\mathrm{NiO}$ monolayer is different from those of thicker films. These issues lead to unique features in photoelectron spectra $[55,57]$ and to a high 

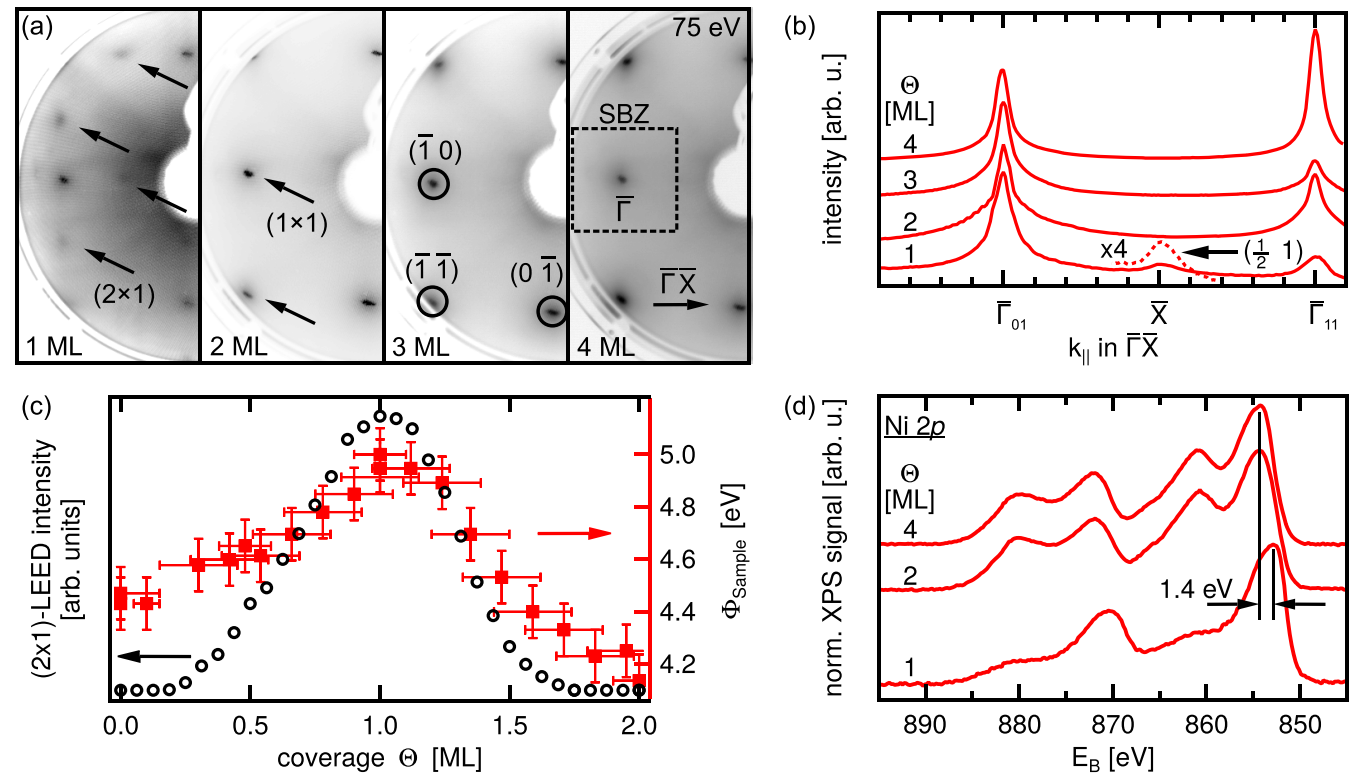

FIG. 2. (a) Diffraction patterns of annealed NiO films on $\mathrm{Ag}(001)$ with thicknesses of $1-4 \mathrm{ML}$ at 75 eV electron energy. The appearing diffraction spots, the surface Brillouin zone (SBZ), as well as the $\overline{\Gamma X}$ direction are denoted. (b) Intensity profiles across the LEED images along the $\overline{\Gamma X}$ direction of the SBZ. (c) LEED intensity of the $(2 \times 1)$ spots (black open circles, left scale) and work function $\Phi$ as measured by UPS (red solid squares, right scale), respectively, vs NiO coverage for an as-prepared sample. (d) XP spectra of the Ni2 $p$ core levels for 1,2 , and 4 ML NiO/Ag(001). All spectra are normalized to their maximum and shifted in intensity for the sake of clarity.

work function with respect to other coverages. In Fig. 2(d) $\mathrm{XP}$ spectra of the Ni $2 p$ core level are depicted for $\mathrm{NiO}$ films from 1-4 ML thickness. While the spectra for 2 and $4 \mathrm{ML}$ are similar and even bulklike in shape [58,59], the spectrum for 1 $\mathrm{ML} \mathrm{NiO/Ag}(001)$ differs significantly: The maximum of the Ni $2 p_{3 / 2}$ peak appears with a difference of $1.4 \mathrm{eV}$ at lower binding energy, and the satellite structure is differently shaped in comparison to the spectra for higher coverages. This is in accordance with findings in the literature $[55,57]$ and can be taken as a marker for this thickness.

In Fig. 2(c) the work function is plotted as function of the oxide film thickness. For $1 \mathrm{ML}$ it shows a characteristic maximum of $\Phi_{\mathrm{ML}} \sim 5.0 \mathrm{eV}$. This value differs strongly from that of clean $\operatorname{Ag}(001)\left(\Phi_{\mathrm{Ag}} \sim 4.5 \mathrm{eV}\right)$ and these of thicker films $\left(\Phi_{\mathrm{NiO}} \sim 4.2-4.4 \mathrm{eV}\right)$. Therefore, one can clearly identify one monolayer also on the basis of work function measurements.

\section{RESULTS}

In Fig. $32 \mathrm{PPE}$ spectra for two different preparations of $2 \mathrm{ML}$ $\mathrm{NiO} / \mathrm{Ag}(001)$ are depicted for fixed time delays $\Delta t$ between pump (UV) and probe (IR) pulse. The spectra of Fig. 3(a) were obtained directly after film preparation at room temperature (RT) using photon energies of 4.27 and $1.68 \mathrm{eV}$ for pump and probe, respectively. Four different features are identified in the spectra for time delays of $\Delta t=0$ and $115 \mathrm{fs}$. Long-living states dominate the spectrum at $\Delta t=115 \mathrm{fs}$ and are marked with 1, 2, and 3 in Fig. 3(b). These features correspond to energies of 3.78, 4.04, and $4.14 \mathrm{eV}$ (each $\pm 0.05 \mathrm{eV}$ ) above $E_{F}$ in Fig. 3(a). A fourth short-living feature, tagged with B, is located at $3.91 \mathrm{eV}$.

The spectra of Fig. 3(b) were measured with photon energies of 4.17 (pump) and $1.72 \mathrm{eV}$ (probe beam) after annealing the sample at $540 \mathrm{~K}$. Again, the spectra can be described by four different states at energies of 3.75, 4.05, 4.14, and $3.90 \mathrm{eV}$ (B). In contrast to the measurement directly after RT preparation, the spectra are dominated by feature 1 for both time delays $\Delta t$. Due to the reduced pump photon energy of $4.17 \mathrm{eV}$, state 3 is hardly populated and almost not visible in the spectra.

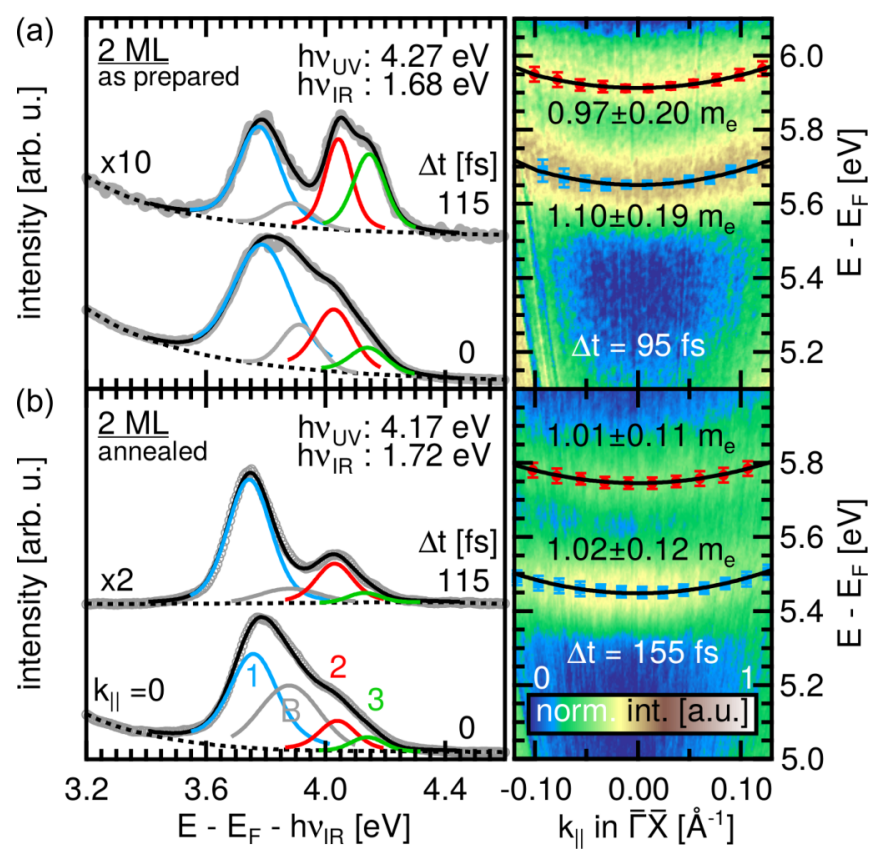

FIG. 3. $2 \mathrm{ML} \mathrm{NiO/Ag(001):} 2 \mathrm{PPE}$ spectra at $k_{\|}=0$ (left panel) and momentum-resolved data (right panel). The data have been measured (a) directly after film preparation and (b) after annealing at $540 \mathrm{~K}$ for time delays of $\Delta t=0$ and $\Delta t=115 \mathrm{fs}$, respectively. The as-prepared (annealed) sample was pumped with $h \nu_{\mathrm{UV}}=4.27 \mathrm{eV}$ $(4.17 \mathrm{eV})$ and probed with $h v_{\mathrm{IR}}=1.68 \mathrm{eV}(1.72 \mathrm{eV})$. 


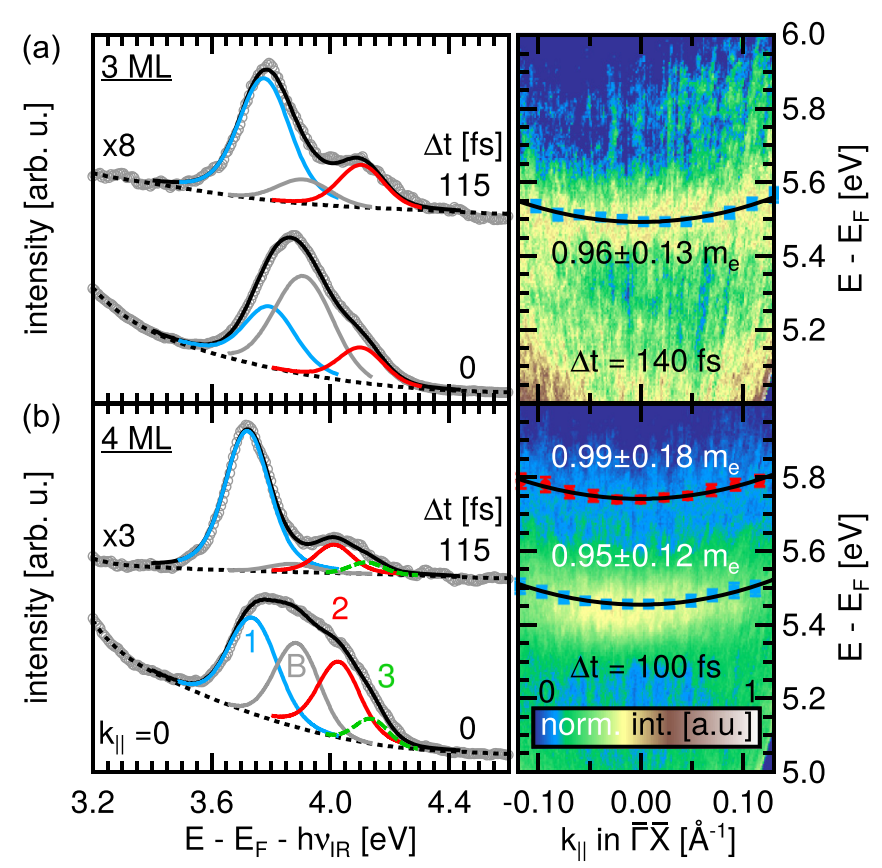

FIG. 4. 2PPE spectra for $k_{\|}=0$ (left panel) and momentumresolved data (right panel) for $3 \mathrm{ML}$ (a) and $4 \mathrm{ML}$ (b) NiO/Ag(001), pumped with $h \nu_{\mathrm{UV}}=4.17 \mathrm{eV}$ and probed with $h \nu_{\mathrm{IR}}=1.72 \mathrm{eV}$ at different time delays $\Delta t$.

Momentum-resolved data (right panel in Fig. 3) reveal a dispersion of the states 1 and 2 with effective masses for the asprepared (annealed) sample of $m_{\mathrm{eff}}=1.1$ (1.02) $m_{e}$ for feature 1 and 0.97 (1.01) $m_{e}$ for feature 2, respectively. In other words, both states show a free-electron-like dispersion parallel to the sample surface.

For 3 and $4 \mathrm{ML} \mathrm{NiO/Ag(001),} \mathrm{similar} \mathrm{unoccupied} \mathrm{states} \mathrm{are}$ found (Fig. 4). The energies of these features are 3.79, 4.11 for the first and second state and $3.90 \mathrm{eV}$ (B) for $3 \mathrm{ML}$ [60]. They shift to $3.73,4.02(1,2)$ and $3.89 \mathrm{eV}$ (B) for $4 \mathrm{ML}$ (each \pm 0.05 $\mathrm{eV})$. The energetic positions of these unoccupied states differ only slightly from those of the $2 \mathrm{ML}$ states. The features show a dispersion (right panel in Fig. 4) with effective masses $m_{\text {eff }}$ of $0.96,0.95$ (feature 1), and $0.99 m_{e}$ (feature 2). In addition, one can again recognize a short-living feature $\mathrm{B}$ as has been seen for $2 \mathrm{ML}$.

In Table I these data are summarized together with the sample work functions $\Phi$ obtained by UPS. For the as-prepared bilayer, the work function $\Phi$ amounts to $4.18 \mathrm{eV}$ and is about $250 \mathrm{meV}$ lower than for the annealed $\mathrm{NiO}$ films with 2-4 ML thickness. This difference is explained according to the Smoluchowski effect [61] by a higher step density for the as-prepared $\mathrm{NiO}$ film.

The analysis of the 2PPE data was done as follows. The raw data (CCD images) were measured by tuning the temporal overlap between pump (UV) and probe (IR) beam. From the raw CCD detector images energy-resolved intensity profiles around a photoemission polar angle of 0 deg with an angular width of $4 \mathrm{deg}$ were extracted and used as spectra for the analysis. For delays far from the temporal overlap of both laser pulses, a spectrum only consists of the single contributions of the UV and the IR beam, respectively. These so called single

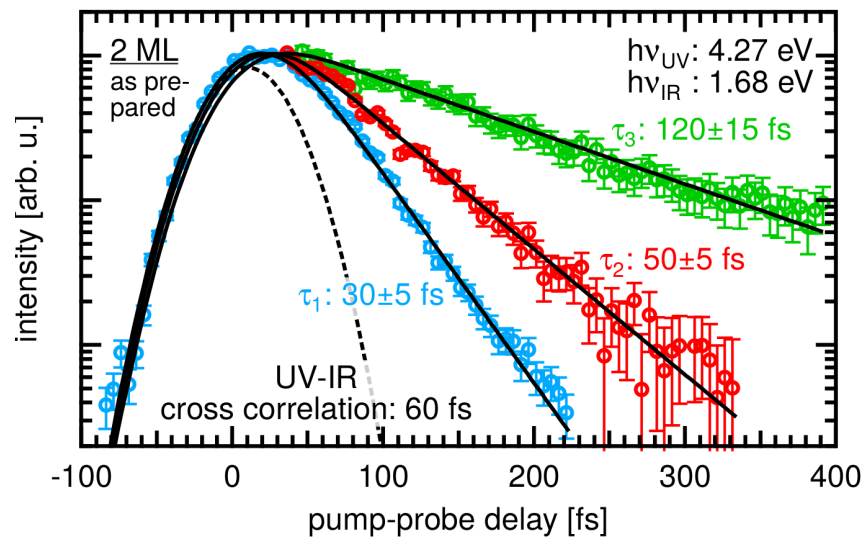

FIG. 5. Time-dependent 2PPE intensities (data points) of the features 1 (blue, bottom), 2 (red, middle), and 3 (green, top) for the as-prepare $2 \mathrm{ML} \mathrm{NiO} / \mathrm{Ag}(001)$. The intensities are described by rate equations resulting in the decay curves (black solid lines) which yield the denoted well-defined lifetimes. The dashed black line represents the cross correlation between UV and IR pulses.

color contributions were subtracted from each spectrum and for each time delay in a first step. Second, the photoemission background due to secondary electron processes was subtracted (black dotted lines in Figs. 3 and 4). The double-corrected spectra were then fitted using Voigt profiles for all time delays between UV and IR, generating the time-dependent intensity of each single Voigt profile (data points in Figs. 5 and 6).

In Fig. 5 time-dependent 2PPE intensities for the features 1-3 are depicted for the as-prepared $2 \mathrm{ML} \mathrm{NiO(001)} \mathrm{film.}$ The data points are fitted using a rate equation approach (black solid lines). For comparison, the cross correlation curve between pump and probe pulse which has been determined simultaneously and which has a full width at half maximum (FWHM) of $60 \mathrm{fs}$ is also included in Fig. 5. The solid lines describe the experimental data well over two and a half orders

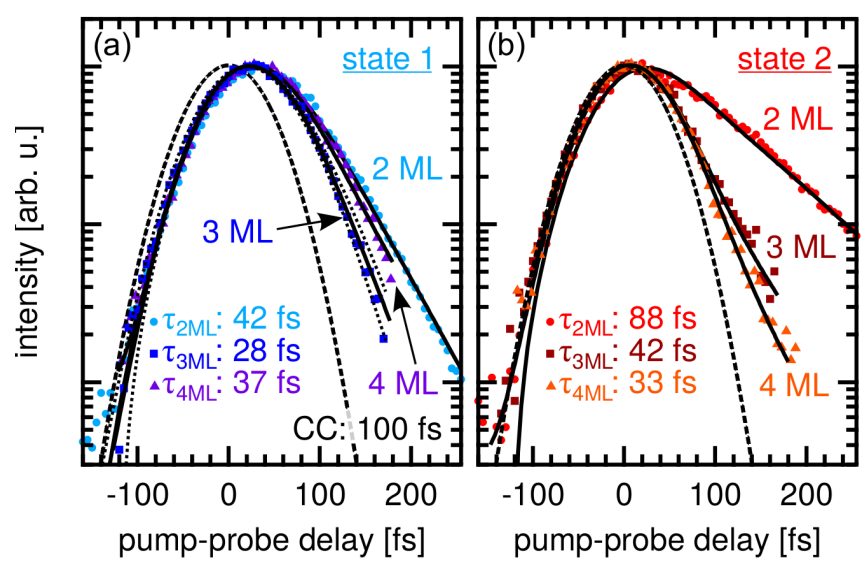

FIG. 6. Time-dependent 2PPE intensities (data points) of (a) state 1 (blue colors) and (b) state 2 (red colors) of annealed 2-4 $\mathrm{ML} \mathrm{NiO/Ag(001).} \mathrm{For} \mathrm{comparison,} \mathrm{the} \mathrm{cross} \mathrm{correlation} \mathrm{traces} \mathrm{are}$ depicted as dashed black lines. The dotted black lines in (a) are data fits resulting from -5 fs shorter and +5 fs longer lifetimes as compared to the best data fit for $3 \mathrm{ML}$ (black solid line). 
TABLE I. Intermediate state energies $E_{\text {int }}$, binding energies $E_{B}$, effective masses $m_{\text {eff }}$, and lifetimes $\tau$ of the first three image potential states for $\mathrm{NiO}(001)$ ultrathin films on $\mathrm{Ag}(001)$ with thicknesses between 2 and $4 \mathrm{ML}$. For comparison, data for the IPS on bare $\mathrm{Ag}(001)$ [47] are included (marked $0 \mathrm{ML}$ ). The second column gives the work functions $\Phi$ as measured by UPS. The uncertainties amount to \pm 50 meV for the given energies.

\begin{tabular}{|c|c|c|c|c|c|c|c|c|c|c|c|c|}
\hline \multirow[b]{2}{*}{$\begin{array}{l}\text { Coverage } \\
\text { (ML) }\end{array}$} & \multirow[b]{2}{*}{$\begin{array}{c}\Phi \\
(\mathrm{eV})\end{array}$} & \multicolumn{4}{|c|}{ State 1} & \multicolumn{4}{|c|}{ State 2} & \multicolumn{3}{|c|}{ State 3} \\
\hline & & $\begin{array}{c}E_{\text {int }} \\
(\mathrm{eV})\end{array}$ & $\begin{array}{c}E_{B} \\
(\mathrm{meV})\end{array}$ & $\begin{array}{l}m_{\mathrm{eff}} \\
\left(m_{e}\right)\end{array}$ & $\begin{array}{c}\tau \\
(\mathrm{fs})\end{array}$ & $\begin{array}{c}E_{\text {int }} \\
(\mathrm{eV})\end{array}$ & $\begin{array}{c}E_{B} \\
(\mathrm{meV})\end{array}$ & $\begin{array}{l}m_{\text {eff }} \\
\left(m_{e}\right)\end{array}$ & $\begin{array}{c}\tau \\
(\mathrm{fs})\end{array}$ & $\begin{array}{c}E_{\text {int }} \\
(\mathrm{eV})\end{array}$ & $\begin{array}{c}E_{B} \\
(\mathrm{meV})\end{array}$ & $\begin{array}{c}\tau \\
\text { (fs) }\end{array}$ \\
\hline 0 [47] & 4.43 & & 561 & & 57 & & 170 & & 165 & & 72 & 380 \\
\hline $2^{\mathrm{a}}$ & 4.18 & 3.78 & 400 & $1.10 \pm 0.19$ & $30 \pm 5$ & 4.04 & 120 & $0.95 \pm 0.20$ & $50 \pm 5$ & 4.14 & 40 & $120 \pm 15$ \\
\hline $2^{\mathrm{b}}$ & 4.44 & 3.75 & 690 & $1.02 \pm 0.12$ & $42 \pm 6$ & 4.05 & 390 & $1.01 \pm 0.11$ & $88 \pm 18$ & 4.14 & & \\
\hline $3^{\mathrm{b}}$ & 4.43 & 3.79 & 640 & $0.96 \pm 0.13$ & $28 \pm 5$ & 4.11 & 320 & & $42 \pm 14$ & & & \\
\hline $4^{\mathrm{b}}$ & 4.41 & 3.73 & 680 & $0.95 \pm 0.12$ & $37 \pm 8$ & 4.02 & 390 & $0.99 \pm 0.18$ & $33 \pm 13$ & (4.14) & & \\
\hline
\end{tabular}

\footnotetext{
${ }^{\text {a As-prepared. }}$.

${ }^{\mathrm{b}}$ Annealed to $540 \mathrm{~K}$.
}

of magnitude in intensity and yield lifetimes of $30 \pm 5,50 \pm 5$, and $120 \pm 15$ fs for the three states.

For the annealed $2 \mathrm{ML}$ film, the lifetimes (Fig. 6) differ significantly from those of the as-prepared film. The lifetime of feature 1 is about 12 fs longer ( $42 \pm 6 \mathrm{fs}$ ), whereas the lifetime of feature 2 has increased by 38 fs (to $88 \pm 18 \mathrm{fs}$ ). In Fig. 6 a comparison of the time-resolved 2PPE data for 2, 3, and $4 \mathrm{ML}$ (all annealed to $540 \mathrm{~K}$ ) is presented. The fits yield lifetimes of $28 \pm 5$ and $37 \pm 8$ fs for state 1 of 3 and 4 ML. The lifetimes of state 2 for 3 and $4 \mathrm{ML}$ amount to $42 \pm 14$ and $33 \pm 13 \mathrm{fs}$, respectively.

Despite a relatively large pump-probe cross correlation of $100 \mathrm{fs}$ (cf. Fig. 6), the lifetimes of the unoccupied states can be determined with an accuracy down to \pm 5 fs due to the in situ determination of time zero and cross correlation. During the fitting process, the uncertainties of the time zero and of the cross correlation were included as constraints [48]. In Fig. 6(a), the comparison of lifetime fits with $28 \mathrm{fs}$ (solid line) and with $5 \mathrm{fs}$ shorter as well as longer lifetimes (dotted lines) demonstrates the lifetime accuracy which is similar to previous studies [4].

The lifetime of feature B at about $3.9 \mathrm{eV}$ (cf. Figs. 3 and 4) has been found to be lower than $15 \mathrm{fs}$. A discussion of this fast-decaying state is carried out at the end of the following section.

\section{DISCUSSION}

Figure 1 depicts the projected band structures of $\operatorname{Ag}(001)$ and $\mathrm{NiO}(001)$ as well as their alignments. For bare $\mathrm{Ag}(001)$, momentum-resolved 2PPE data are shown in Fig. 1(a). One can recognize the strongly dispersing unoccupied $s p$ bulk bands which extend at $k_{\|}=0$ to $\sim 2 \mathrm{eV}$ above $E_{F}$. It defines the lower edge of the $\operatorname{Ag}(001)$ band gap at the $\bar{\Gamma}$ point which reaches well above the vacuum level $[41,42,62]$. Additionally, the first two image potential states at 3.89 and $4.25 \mathrm{eV}$ above $E_{F}$ are well reproduced in good agreement with recent studies $[2,4,47,63]$.

The electronic structure of $\mathrm{NiO}(001)$ and $\mathrm{NiO}(001) / \mathrm{Ag}(001)$ in the energy region near $E_{F}$ has been investigated by photoemission and inverse photoemission spectroscopy [38,40,64-66]. In these studies, the valence band maximum has been found at 1.5-2 eV below $E_{F}$ for bulk $\mathrm{NiO}$ which fits to our UPS data (not shown here). On the other hand, the onset of inverse photoemission features has been assigned to the $\mathrm{NiO}$ conduction band (CB) which is formed by the Ni3d upper Hubbard band. This defines the $\sim 4 \mathrm{eV}$ fundamental band gap of bulk NiO. Theoretical studies of the $\mathrm{NiO}$ band structure support this assignment and emphasize the strong effect of electron correlation [45,67-69]. The surface-projected band structure for bulk $\mathrm{NiO}$ along the $\bar{\Gamma} X$ direction as adapted from Schrön et al. [45] and shifted according to the experimentally determined $\mathrm{NiO}$ band gap is sketched in Fig. 1(c). The NiO CB starts at $2.5 \mathrm{eV}$ and is formed by localized $\mathrm{Ni} 3 d$ states, whereas O-derived states form stronger dispersing $s p$ bands centered around $\bar{\Gamma}$ at higher energies [45].

All experiments described here use laser pulses with photon energies of 4.2-4.3 eV for the primary photoexcitation process. In principle, these photon energies enable excitation across the band gap of $\mathrm{NiO}$. Such excited electrons could populate states up to $2.7 \mathrm{eV}$ above $E_{F}$ only due to the location of the valence band maximum at $1.5 \mathrm{eV}$ below $E_{F}$. In contrast, the photoelectrons close to the vacuum level that are the subject in the present paper are at higher energies and are, therefore, pumped from metallic substrate states in the energy region slightly below the Fermi level as indicated by the blue arrow in Fig. 1(b). Note that the photon energies of the infrared probe pulses are too low for any primary photoexcitation.

The position and the dispersion of the experimentally observed unoccupied states for 2-4 ML NiO/Ag(001) are depicted in Fig. 1(c) as red parabolae. For comparison, the calculated states of the $s p$-derived bands that exhibit surface state or surface resonance character are indicated on the lefthand side [45]. They disperse with effective masses of about $0.6 m_{e}$ (orange dashed lines) and nearly $1 m_{e}$ (blue dashed dotted lines) [45]. Inside the charge-transfer gap of $\mathrm{NiO}$, a barely dispersing surface state is located around $2 \mathrm{eV}$ above $E_{F}$ (black line).

In the 2PPE experiments, we find a series of three unoccupied states converging towards the vacuum level in the energy range between 3.5 and $4.4 \mathrm{eV}$ above $E_{F}$. In Table I their intermediate state energies $E_{\text {int }}$ with respect to the Fermi level, binding energies $E_{B}$ with respect to the vacuum level, effective masses $m_{\text {eff }}$, and lifetimes $\tau$ are summarized for 2, 3, and $4 \mathrm{ML}$ of $\mathrm{NiO}(001)$ on $\mathrm{Ag}(001)$. Data for the work function (adopted by UPS) and the IPS on bare $\mathrm{Ag}(001)$ (marked $0 \mathrm{ML}$ coverage) [47] are included for comparison. 
In the following we discuss the three unoccupied states in three steps. First, we rule out a possible observation of pure unoccupied NiO bulk states. Second, we assign them to image potential resonances. The discussion is, finally, followed up by comments on the interaction of image potential and quantumwell states that develop inside the $\mathrm{NiO}$ film.

(i) $\mathrm{NiO}$ conduction band states are expected in the energy range of the observed unoccupied states [cf. Fig. 1(c)]. In fact, the theoretical study of Schrön et al. predicts flat unoccupied $d$ bands and stronger dispersing unoccupied $s p$ bands. While the band dispersions are theoretically described well, the absolute energies of the calculated unoccupied states have to be taken with care. Besides a $d_{z^{2}}$ derived unoccupied surface state around $2 \mathrm{eV}$, distinct $s p$-derived $\mathrm{NiO}(001)$ states at 3.0 and 3.70 $\mathrm{eV}\left(m_{\mathrm{eff}} \sim 0.6 m_{e}\right)$ and at 4.67 and $5.14 \mathrm{eV}\left(m_{\mathrm{eff}} \sim 1.0 m_{e}\right)$, respectively, are marked as dotted and dashed-dotted lines in Fig. 1(c). These states have an increased probability density at the surface. According to the excitation scheme depicted in Fig. 1(b) we have to consider a direct population of such $\mathrm{NiO}$ CB states by the pump pulse, i.e., a pure $d$ or $s p$ character of the found states.

All investigated 2PPE features show a dispersion with effective masses of about $1 m_{e}$. Therefore, one can directly exclude a $d$-derived character of these states. In the same way, a pure $s p$ character is also less likely: The observed features occur in the low energy range of the $s p$-derived $\mathrm{NiO} \mathrm{CB}$. Here the theoretically predicted CB states at 3.0 and $3.7 \mathrm{eV}$ above the Fermi level could be addressed by the UV pump pulse. However, these $\mathrm{NiO} s p$ states disperse with effective masses of $\sim 0.6$ along the $\overline{\Gamma X}$ direction which are only one half of that of the investigated states. On the other hand, the theoretically predicted surface resonances at 4.67 and $5.14 \mathrm{eV}$ [blue dashed-dotted in Fig. 1(c)] show effective masses of $1 m_{e}$. However, their energies are well above the bottom of the $s p$-derived $\mathrm{CB}$ and are located to high with respect to the experimentally observed states.

As a consequence, the found 2PPE feature have to be characterized differently.

(ii) The image potential in front of a dielectric substrate is modified as compared with the situation in front of a metallic substrate by a factor $\beta$ :

$$
V(z)=-\frac{e^{2}}{4 \pi \varepsilon_{0}} \frac{\beta}{4 z}, \quad \beta=\frac{\varepsilon-1}{\varepsilon+1},
$$

where $\varepsilon$ denotes the static dielectric constant and $z$ is the distance from the dielectric surface. For an ultrathin film on a metal substrate, the finite thickness of the dielectric layer and the metallic response have additionally to be taken into account as discussed previously [70,71]

$$
V(z)=-\frac{e^{2}}{4 \pi \varepsilon_{0}}\left[\frac{\beta}{4 z}-\frac{1-\beta^{2}}{4 \beta} \sum_{k=1}^{\infty} \frac{(-\beta)^{k}}{z+k d}\right],
$$

where $d$ is the thickness of the dielectric layer. The first term of Eq. (2) describes the interaction between an electron and a dielectric layer, whereas the second term contains modifications of the dielectric response due to the metal underneath. For thick films or for $\beta$ close to 1 , the potential in front of the surface is dominated by the image potential of the dielectric. The dielectric constant for $\mathrm{NiO}$ of $\varepsilon=12$ [72] yields $\beta=0.85$ which is already close to one. Therefore, we expect only slightly modified binding energies for the IPS of NiO/Ag(001) as compared to the bare metal [71]. The binding energies $E_{n}$ for a Rydberg-like series of electronic states converging to the vacuum level is given by $E_{n}=0.85 \cdot \beta^{2} m_{\mathrm{eff}} /(n+a)^{2}$, where $n$ is the quantum number of the state and $a$ is the quantum defect. In case of nearly free electrons $\left(m_{\mathrm{eff}}=1\right)$ in front of a metal $(\beta \rightarrow 1)$, the largest possible binding energy for the first (second) IPS amounts to $850(425) \mathrm{meV}$. The here observed binding energies of $640-690 \mathrm{meV}$ for the first peaks as well as $320-390 \mathrm{meV}$ for the second peaks (annealed films) fall therefore well in the energy range of IPS. The assignment of the 2PPE features to IPS is furthermore corroborated by the effective masses of these unoccupied states which are close to $1 m_{e}$. In addition, the lifetimes of energetically higher lying features of 2 and $3 \mathrm{ML} \mathrm{NiO/Ag(001)} \mathrm{are} \mathrm{longer} \mathrm{as} \mathrm{compared}$ to lower lying ones (cf. Table I) following the general trend known from clean metals [73]. All of these findings are typical for IPS and support our interpretation.

Taking the band structure of the underlying $\mathrm{NiO}$ films into account [Fig. 1(b)] one recognizes that all features are in resonance with the $\mathrm{NiO}$ conduction band. Consequently, we have to consider them as image potential resonances (IPR).

As a result of a potentially resonant character the energies with respect to the Fermi level (IPR 1: $3.75 \mathrm{eV}$, IPR 2: $4.07 \mathrm{eV}$, IPR 3: $4.14 \mathrm{eV}$ ) change only slightly with $\mathrm{NiO}$ film thickness. The small variations by $50 \mathrm{meV}$ for IPR 1 and $70 \mathrm{meV}$ for IPR 2 can be explained by small differences in the strength of coupling.

(iii) The investigated system $\mathrm{Ag}(001)-\mathrm{NiO}(001)$-vacuum contains two interfaces: between the substrate and the dielectric film and between the film and the vacuum, respectively [Fig. 1(b)]. Energy barriers are formed on both sides of the $\mathrm{NiO}$ film due to the large energy gap between 2 and $7 \mathrm{eV}$ in the band structure of $\operatorname{Ag}(001)$ on the one side and the vacuum on the other side. This leads to a break-up of the NiO CB and a formation of quantum-well states (QWS) inside the oxide film. Therefore, we have to consider a coupling of the IPS with such quantum-well states.

The interaction of IPS and QWS has been investigated previously [10,12,15-17,27]. Resonant coupling between IPS and QWS would lead to a lowering of the lifetime and, in accordance, to a significant increase of the binding energy of the IPR as has been investigated on rare gas adlayers on metal surfaces $[16,17]$. The coupling condition changes with increasing adlayer thickness according to the change of the width of the quantum well. This should be reflected in the lifetime and binding energy behavior of the coupled image potential states $[15,16]$. Furthermore, resonant coupling can even lead to a complete transition of states having image potential character to states with pure quantum well character. This is accompanied by an extensive broadening of the peak widths due to efficient coupling to the bulk [11,27].

For $\mathrm{NiO}(001)$ on $\mathrm{Ag}(001)$ we find an oscillatory behavior of the lifetime of the first IPR which is similar to, e.g., image potential states on $\mathrm{Xe} / \mathrm{Ag}(111)$ [16]. Moreover, the lifetime of the second IPR decreases monotonically with increasing $\mathrm{NiO}$ film thickness comparable to 2PPE measurements on $\mathrm{Xe} / \mathrm{Cu}(100)$ and $\mathrm{Kr} / \mathrm{Cu}(100)$ [17]. Hence, our findings could be interpreted by the formation of quantum-well resonances 
(a) at the energy of the first IPR for $3 \mathrm{ML}$ and (b) at the energy of the second IPR for NiO film thicknesses $\geqslant 4$ ML. However, the typical increase of the binding energies with increasing lifetime does not occur for the first nor for the second IPR (Table I). On the contrary, the binding energy decreases at 3 ML, whereas is nearly constant for the other $\mathrm{NiO}$ coverages without noticeable dependence on the lifetime. In addition, no significant broadening of the peaks is visible from the spectra in Figs. 3 and 4. Therefore, we assign the series of 2PPE features to image potential states that are weakly coupled to $\mathrm{NiO}$ bulk states.

The wave functions of the unoccupied states couple to states of the unoccupied $s p$-derived band of the $\mathrm{NiO}$ film which can be read from their energetic position [Fig. 1(c)] as well as their persistent dispersion with $m_{e} \sim 1$. The resonance between IP states and the $\mathrm{NiO} \mathrm{CB}$ leads to a shift of their probability density towards the sample surface [17]. The resulting enhanced penetration of the wave function into the substrate explains the observed lifetime reduction from $57 \mathrm{fs}$ (165 fs) of the $n=1(n=2)$ IPS of bare $\operatorname{Ag}(001)$ to $30-40$ fs for the first IPR and $<90$ fs for the second IPR of annealed 2-4 ML NiO/Ag(001) due to an additional decay channel for resonant charge transfer into the bulk.

The electronic structure of the $\mathrm{NiO}(001) / \mathrm{Ag}(001)$ thin film system may differ from the bulk band structure depicted in Fig. 1(c) and is varying with increasing film thickness, e.g., by formation of QW states. As a consequence, the coupling conditions between IPS and $s p$-derived states also vary. For example, the remarkably shorter lifetime of 28 fs for the first IPS of $3 \mathrm{ML} \mathrm{NiO/Ag(001)} \mathrm{points} \mathrm{towards} \mathrm{a} \mathrm{stronger} \mathrm{coupling}$ of IPR to $s p$-derived states that evolve at this specific thickness. In a similar way, the different IPR lifetimes for the $\mathrm{NiO}$ layers are explained by modifications of the underlying electronic structure and, therefore, different coupling conditions. The lifetime of electrons from the second IPR decreases monotonically with increasing film thickness. For $4 \mathrm{ML}$, the lifetime of the second IPR electrons is even shorter than for electrons excited into the accordingly first IPR. This is explained by matching resonance conditions for the second IPR at 4 ML film thickness.

To promote our interpretations, detailed theoretical calculations on the layer-resolved band structure of the $\mathrm{NiO}$ films would be desirable. In addition, experiments on thicker $\mathrm{NiO}$ films would be helpful to get the transition from a thin film to a bulklike behavior.

One can recognize distinct variations between the data of the as-prepared $2 \mathrm{ML}$ and the annealed $2 \mathrm{ML} \mathrm{NiO}$ films. According to the above interpretation of image-potential derived states, these differences can be assigned to the quality of the $\mathrm{NiO}$ films: The observed states for the as-prepared and the annealed $2 \mathrm{ML} \mathrm{NiO}$ films on $\mathrm{Ag}(001)$ differ only slightly in energy with respect to $E_{F}$ (cf. Table I). In contrast, the binding energies of the features of the as-prepared sample as well as the work function are approximately $250 \mathrm{meV}$ lower as compared to the annealed samples. This is interpreted within the concept of the local work function [10]. Work function measurements average over a large sample area containing flat $\mathrm{NiO}$ terraces as well as stepped $\mathrm{NiO}$ regions, resulting in a measured global work function. Electrons in an image potential state are located in front of the flat $\mathrm{NiO}$ terraces only. Therefore, they are affected by the local work function of the flat $\mathrm{NiO}$ regions. Since the energies of the IPR do not change for the as-prepared and annealed $\mathrm{NiO}$ bilayer, the local work function does not change for both preparations. Due to an enhanced film quality upon annealing the $\mathrm{NiO}$ bilayer, the global work function is close to that of the flat terraces for the annealed $\mathrm{NiO}$ bilayer. Hence, the correct binding energy of the IPR is reflected by the annealed bilayer.

The 12 fs shorter lifetime for the as-prepared 2 ML film in comparison to the film which has been annealed to $540 \mathrm{~K}$ demonstrates the influence of film imperfections on the IPR lifetime. As known from other systems, the electron dynamics strongly depend on defect and step densities [2,74-76]. These densities lead to a nonmomentum conserving scattering and, therefore, open up additional relaxation channels. For the annealed oxide films, the quality of the layers of different thicknesses as determined by the sharpness of the LEED spots [cf. Fig. 2(a)] is comparable. The film quality is significantly higher as compared to the as-prepared $2 \mathrm{ML}$ film. Therefore, the IPR lifetimes for all annealed films should be less limited by surface imperfections. Usually, the second IPS is even more sensitive to the film quality as compared to the first IPS due to its longer lifetime and due to the possibility of nonmomentum conserving scattering into the first IPS. Indeed, the lifetime of the as-prepared $2 \mathrm{ML}$ film is reduced to 50 in comparison to 88 fs for the annealed film.

In addition to the series of IPR, a short-living ( $<15 \mathrm{fs}$ ) unoccupied state $\mathrm{B}$ at about $3.9 \mathrm{eV}$ above $E_{F}$ has been observed for 2-4 ML NiO/Ag(001) (Figs. 3 and 4). Despite its origin is not yet fully clear, some statements regarding the character of this unoccupied state can be given. It is obvious that the intermediate energy of state B nearly coincides with that found for the $n=1$ IPS of the clean $\operatorname{Ag}(001)$ substrate $[3.89 \mathrm{eV}$, cf. Fig. 1(a)]. Therefore, it is most likely that feature B is a signal resulting from the $n=1$ IPS of the silver substrate which occurs above remaining bare Ag patches at the sample surface. The substantially shorter lifetime of state B (max. $26 \%$ compared to the $n=1$ IPS of clean $\mathrm{Ag}(001)$ ) can be explained by enhanced scattering probability at residual $\mathrm{Ni}$ or $\mathrm{O}$ sites at the Ag patches. However, due to its energy position and its ultrashort lifetime, feature B can also be a candidate for the directly populated unoccupied O-derived $s p$ state as mentioned above. The decay of such a bulklike state is much faster due to enhanced coupling of its wave function to the bulk compared to the spatially more decoupled image potential states.

\section{CONCLUSIONS}

We have investigated a series of unoccupied states for $\mathrm{NiO}(001)$ ultrathin films of 2-4 ML on $\mathrm{Ag}(001)$ in the energy range between 3.5 and $4.4 \mathrm{eV}$ above $E_{F}$ by means of angleand time-resolved 2PPE. Regarding their energy position, their parabolic dispersion, and their lifetime behavior, they are assigned to image potential states resonant with $\mathrm{NiO}$ conduction band states. For the first IPR the lifetimes show an oscillatory behavior and differ only slightly for annealed $\mathrm{NiO}$ films with 2-4 ML thickness. In contrast, the lifetimes of the second IPR decrease with increasing film thickness from 88 ( $2 \mathrm{ML}$ ) to $33 \mathrm{fs}$ ( $4 \mathrm{ML}$ ). This is explained by the presence of $s p$-derived $\mathrm{NiO}$ states in the energy range of the IPS which 
lead to an enhanced layer-dependent coupling between $\mathrm{NiO}$ and IP states with increasing film thickness and thus to a faster decay compared to bare $\operatorname{Ag}(001)$. Additionally, the lifetime of the IPR depends strongly on the film quality as is demonstrated for a $2 \mathrm{ML} \mathrm{NiO}(001)$ film prepared at RT. Without annealing, the NiO bilayer yields significantly shorter lifetimes of the first and second IPR in comparison to an annealed film.

\section{ACKNOWLEDGMENTS}

The authors thank Ralf Kulla for generous technical assistance and Cheng-Tien Chiang for fruitful discussions. Financial support by the Deutsche Forschungsgemeinschaft via SFB 762 is gratefully acknowledged. K.G. thanks the International Max Planck Research School for Science and Technology of Nanostructures for funding.
[1] K. Giesen, F. Hage, F. J. Himpsel, H. J. Riess, and W. Steinmann, Two-Photon Photoemission via Image-Potential States, Phys. Rev. Lett. 55, 300 (1985).

[2] S. Schuppler, N. Fischer, T. Fauster, and W. Steinmann, Bichromatic two-photon photoemission spectroscopy of image potential states on $\operatorname{Ag}(100)$, Appl. Phys. A 51, 322 (1990).

[3] R. W. Schoenlein, J. G. Fujimoto, G. L. Eesley, and W. Capehart, Femtosecond photoemission studies of image potential and electron dynamics in metals, in Laser Optics of Condensed Matter, edited by E. Garmire, A. Maradudin, and K. Rebane (Springer, New York, 1991), pp. 71-82.

[4] I. L. Shumay, U. Höfer, Ch. Reuß, U. Thomann, W. Wallauer, and T. Fauster, Lifetimes of image-potential states on $\mathrm{Cu}(100)$ and $\operatorname{Ag}(100)$ measured by femtosecond time-resolved two-photon photoemission, Phys. Rev. B 58, 13974 (1998).

[5] A. Schäfer, I. L. Shumay, M. Wiets, M. Weinelt, T. Fauster, E. V. Chulkov, V. M. Silkin, and P. M. Echenique, Lifetimes of unoccupied surface states on Pd(111), Phys. Rev. B 61, 13159 (2000).

[6] S. Link, H. A. Dürr, and W. Eberhardt, Lifetimes of imagepotential states on the $\mathrm{Pt}(111)$ surface probed by time-resolved two-photon photoemission spectroscopy, Appl. Phys. A 71, 525 (2000).

[7] M. Marks, C. H. Schwalb, K. Schubert, J. Güdde, and U. Höfer, Quantum-beat spectroscopy of image-potential resonances, Phys. Rev. B 84, 245402 (2011).

[8] M. Winter, E. V. Chulkov, and U. Höfer, Trapping of ImagePotential Resonances on a Free-Electron-like Surface, Phys. Rev. Lett. 107, 236801 (2011).

[9] X. Cui, C. Wang, A. Argondizzo, S. Garrett-Roe, B. Gumhalter, and H. Petek, Transient excitons at metal surfaces, Nat. Phys. 10, 505 (2014).

[10] R. Fischer, S. Schuppler, N. Fischer, T. Fauster, and W. Steinmann, Image States and Local Work Function for $\mathrm{Ag} / \mathrm{Pd}(111)$, Phys. Rev. Lett. 70, 654 (1993).

[11] R. Fischer and T. Fauster, Coupling of image states to quantumwell states for $\mathrm{Au}$ on $\mathrm{Pd}(111)$, Phys. Rev. B 51, 7112 (1995).

[12] T. Fauster and W. Steinmann, Two-photon photoemission spectroscopy of image states, in Electromagnetic Waves: Recent Developments in Research, edited by P. Halevi (Elsevier, Amsterdam, 1995).

[13] D. F. Padowitz, W. R. Merry, R. E. Jordan, and C. B. Harris, Two-Photon Photoemission as a Probe of Electron Interactions with Atomically Thin Dielectric Films on Metal Surfaces, Phys. Rev. Lett. 69, 3583 (1992).

[14] M. Wolf, E. Knoesel, and T. Hertel, Ultrafast dynamics of electrons in image-potential states on clean and Xe-covered Cu(111), Phys. Rev. B 54, R5295 (1996).
[15] J. D. McNeill, R. L. Lingle, R. E. Jordan, D. F. Padowitz, and C. B. Harris, Interfacial quantum well states of $\mathrm{Xe}$ and $\mathrm{Kr}$ adsorbed on Ag(111), J. Chem. Phys. 105, 3883 (1996).

[16] J. D. McNeill, R. L. Lingle, N.-H. Ge, C. M. Wong, R. E. Jordan, and C. B. Harris, Dynamics and Spatial Distribution of Electrons in Quantum Wells at Interfaces Determined by Femtosecond Photoemission Spectroscopy, Phys. Rev. Lett. 79, 4645 (1997).

[17] W. Berthold, F. Rebentrost, P. Feulner, and U. Höfer, Influence of $\mathrm{Ar}, \mathrm{Kr}$, and $\mathrm{Xe}$ layers on the energies and lifetimes of imagepotential states on $\mathrm{Cu}(100)$, Appl. Phys. A 78, 131 (2004).

[18] J. Güdde and U. Höfer, Femtosecond time-resolved studies of image-potential states at surfaces and interfaces of rare-gas adlayers, Prog. Surf. Sci. 80, 49 (2005).

[19] A. Hotzel, Electron dynamics of image potential states in weakly bound adsorbate layers: A short review, Prog. Surf. Sci. 82, 336 (2007).

[20] A. Damm, K. Schubert, J. Güdde, and U. Höfer, Observation of the transition from image-potential states to resonances on argon-covered $\mathrm{Cu}(111)$ and $\mathrm{Ag}(111)$ by time-resolved twophoton photoemission, Phys. Rev. B 80, 205425 (2009).

[21] N.-H. Ge, C. M. Wong, and C. B. Harris, Femtosecond studies of electron dynamics at interfaces, Acc. Chem. Res. 33, 111 (1999).

[22] K. J. Gaffney, A. D. Miller, S. H. Liu, and C. B. Harris, Femtosecond dynamics of electrons photoinjected into organic semiconductors at aromatic-metal interfaces, J. Phys. Chem. B 105, 9031 (2001).

[23] X.-Y. Zhu, Electronic structure and electron dynamics at molecule-metal interfaces: Implications for molecule-based electronics, Surf. Sci. Rep. 56, 1 (2004).

[24] P. S. Kirchmann, P. A. Loukakos, U. Bovensiepen, and M. Wolf, Ultrafast electron dynamics studied with time-resolved two-photon photoemission: Intra- and interband scattering in $\mathrm{C}_{6} \mathrm{~F}_{6} / \mathrm{Cu}(111)$, New J. Phys. 7, 113 (2005).

[25] E. Varene, I. Martin, and P. Tegeder, Optically induced interand intrafacial electron transfer probed by two-photon photoemission: Electronic states of sexithiophene on $\mathrm{Au}(111)$, J. Phys. Chem. Lett. 2, 252 (2011).

[26] M. Shibuta, N. Hirata, R. Matsui, T. Eguchi, and A. Nakajima, Charge separation at the molecular monolayer surface: Observation and control of the dynamics, J. Phys. Chem. Lett. 3, 981 (2012).

[27] J. Zhao, M. Feng, D. B. Dougherty, H. Sun, and H. Petek, Molecular electronic level alignment at weakly coupled organic film/metal interfaces, ACS Nano 8, 10988 (2014).

[28] P. M. Echenique, R. Berndt, E. V. Chulkov, T. Fauster, A. Goldmann, and U. Höfer, Decay of electronic excitations at metal surfaces, Surf. Sci. Rep. 52, 219 (2004). 
[29] S. S. Tsirkin, A. G. Borisov, and E. V. Chulkov, Green's function approach to the lifetimes of image potential resonances at metal surfaces, Phys. Rev. B 88, 035449 (2013).

[30] D. C. Marinica, C. Ramseyer, A. G. Borisov, D. Teillet-Billy, J. P. Gauyacq, W. Berthold, P. Feulner, and U. Höfer, Effect of an Atomically Thin Dielectric Film on the Surface Electron Dynamics: Image-Potential States in the $\mathrm{Ar} / \mathrm{Cu}(100)$ System, Phys. Rev. Lett. 89, 046802 (2002).

[31] P. M. Echenique, J. M. Pitarke, E. V. Chulkov, and A. Rubio, Theory of inelastic lifetimes of low-energy electrons in metals, Chem. Phys. 251, 1 (2000).

[32] K. Onda, B. Li, and H. Petek, Two-photon photoemission spectroscopy of $\mathrm{TiO}_{2}(110)$ surfaces modified by defects and $\mathrm{O}_{2}$ or $\mathrm{H}_{2} \mathrm{O}$ adsorbates, Phys. Rev. B 70, 045415 (2004).

[33] W. A. Tisdale, M. Muntwiler, D. J. Norris, E. S. Aydil, and X.-Y. Zhu, Electron dynamics at the $\mathrm{ZnO}(10 \overline{1} 0)$ surface, J. Phys. Chem. C 112, 14682 (2008).

[34] K. Takahashi, K. Ishibashi, Y. Kurahashi, M. Imamura, J. Azuma, and M. Kamada, Time-resolved two-photon photoemission study of silicon surface at initial stage of oxidation, Appl. Surf. Sci. 267, 154 (2013).

[35] J.-C. Deinert, D. Wegkamp, M. Meyer, C. Richter, M. Wolf, and J. Stähler, Ultrafast Exciton Formation at the $\mathrm{ZnO}(10 \overline{1} 0)$ Surface, Phys. Rev. Lett. 113, 057602 (2014).

[36] A. Argondizzo, X. Cui, C. Wang, H. Sun, H. Shang, J. Zhao, and H. Petek, Ultrafast multiphoton pump-probe photoemission excitation pathways in rutile $\mathrm{TiO}_{2}$ (110), Phys. Rev. B 91, 155429 (2015)

[37] S. Otto and T. Fauster, Two-photon photoemission from $\mathrm{CoO}$ layers on $\operatorname{Ir}(100)$, J. Phys.: Condens. Matter 28, 055001 (2016).

[38] F. Reinert and S. Hüfner, Photoemission spectroscopy-from early days to recent applications, New J. Phys. 7, 97 (2005).

[39] S. Hüfner, Electronic structure of $\mathrm{NiO}$ and related 3d-transitionmetal compounds, Adv. Phys. 43, 183 (2006).

[40] M. Portalupi, L. Duò, G. Isella, R. Bertacco, M. Marcon, and F. Ciccacci, Electronic structure of epitaxial thin $\mathrm{NiO}(100)$ films grown on $\operatorname{Ag}(100)$ : Towards a firm experimental basis, Phys. Rev. B 64, 165402 (2001).

[41] H. Eckardt, L. Fritsche, and J. Noffke, Self-consistent relativistic band structure of the noble metals, J. Phys. F: Metal Phys. 14, 97 (1984).

[42] A. Goldmann, V. Dose, and G. Borstel, Empty electronic states at the (100), (110), and (111) surfaces of nickel, copper, and silver, Phys. Rev. B 32, 1971 (1985).

[43] F. Cinquini, L. Giordano, G. Pacchioni, A. M. Ferrari, C. Pisani, and C. Roetti, Electronic structure of $\mathrm{NiO} / \mathrm{Ag}(100)$ thin films from $D F T+U$ and hybrid functional DFT approaches, Phys. Rev. B 74, 165403 (2006).

[44] I. O. Thomas and A. Fortunelli, Analysis of the electronic structure of ultrathin $\mathrm{NiO} / \mathrm{Ag}(100)$ films, Eur. Phys. J. B 75, 5 (2010).

[45] A. Schrön, M. Granovskij, and F. Bechstedt, Influence of onsite Coulomb interaction $\mathrm{U}$ on properties of $\mathrm{MnO}(001) 2 \times 1$ and $\mathrm{NiO}(001) 2 \times 1$ surfaces, J. Phys.: Condens. Matter 25, 094006 (2013).

[46] A. Höfer, K. Duncker, M. Kiel, S. Förster, and W. Widdra, Laserexcited PEEM using a fully tunable fs-laser system, IBM J. Res. Dev. 55, 1 (2011).
[47] K. Duncker, M. Kiel, and W. Widdra, Momentum-resolved lifetimes of image-potential states on $\operatorname{Ag}(001)$, Surf. Sci. 606, L87 (2012).

[48] See Supplemental Material at http://link.aps.org/supplemental/ 10.1103/PhysRevB.97.085424 for a detailed description of the analysis procedure of the time-resolved 2PPE data .

[49] K. Marre and H. Neddermeyer, Growth of ordered thin films of $\mathrm{NiO}$ on $\mathrm{Ag}(100)$ and $\mathrm{Au}(111)$, Surf. Sci. 287, 995 (1993).

[50] S. Großer, C. Hagendorf, H. Neddermeyer, and W. Widdra, The growth of thin $\mathrm{NiO}$ films on $\mathrm{Ag}(001)$ studied by scanning tunneling microscopy and spectroscopy, Surf. Interface Anal. 40, 1741 (2008).

[51] K. L. Kostov, S. Polzin, S. K. Saha, O. Brovko, V. Stepanyuk, and W. Widdra, Surface-phonon dispersion of a $\mathrm{NiO}(100)$ thin film, Phys. Rev. B 87, 235416 (2013).

[52] T. Bertrams and H. Neddermeyer, Growth of $\mathrm{NiO}(100)$ layers on $\mathrm{Ag}(100)$ : Characterization by scanning tunneling microscopy, $\mathrm{J}$. Vac. Sci. Technol. B 14, 1141 (1996).

[53] I. Sebastian, T. Bertrams, K. Meinel, and H. Neddermeyer, Scanning tunneling microscopy on the growth and structure of $\mathrm{NiO}(100)$ and $\mathrm{CoO}(100)$ thin films, Faraday Discuss. 114, 129 (1999).

[54] A. Dhaka, D. Sander, H.L. Meyerheim, K. Mohseni, E. Soyka, J. Kirschner, W. A. Adeagbo, G. Fischer, A. Ernst, and W. Hergert, Stress and structure at the NiO/Ag(001) interface, Phys. Rev. B 84, 195441 (2011).

[55] M. Caffio, B. Cortigiani, G. Rovida, A. Atrei, and C. Giovanardi, Early stages of $\mathrm{NiO}$ growth on $\mathrm{Ag}(001)$ : A study by LEIS, XPS, and LEED, J. Phys. Chem. B 108, 9919 (2004).

[56] M. Caffio, A. Atrei, B. Cortigiani, and G. Rovida, STM study of the nanostructures prepared by deposition of $\mathrm{NiO}$ on $\mathrm{Ag}(001)$, J. Phys.: Condens. Matter 18, 2379 (2006).

[57] M. Caffio, B. Cortigiani, G. Rovida, A. Atrei, C. Giovanardi, A. di Bona, and S. Valeri, Ultrathin nickel oxide films grown on $\mathrm{Ag}(001)$ : A study by XPS, LEIS and LEED intensity analysis, Surf. Sci. 531, 368 (2003).

[58] D. Alders, F. C. Voogt, T. Hibma, and G. A. Sawatzky, Nonlocal screening effects in $2 p$ x-ray photoemission spectroscopy of NiO(100), Phys. Rev. B 54, 7716 (1996).

[59] L. Sangaletti, L.E. Depero, and F. Parmigiani, On the non-local screening mechanisms in the $2 p$ photoelectron spectra of $\mathrm{NiO}$ and $\mathrm{La}_{2} \mathrm{NiO}_{4}$, Solid State Commun. 103, 421 (1997).

[60] See Supplemental Material at http://link.aps.org/supplemental/ 10.1103/PhysRevB.97.085424 for an alternative version to fit the data for $3 \mathrm{MLNiO} / \mathrm{Ag}(001)$.

[61] R. Smoluchowski, Anisotropy of the electronic work function of metals, Phys. Rev. 60, 661 (1941).

[62] W. Altmann, V. Dose, and A. Goldmann, Momentum-resolved Bremsstrahlung Isochromat Spectroscopy of silver surfaces, Z. Phys. B 65, 171 (1986).

[63] R. W. Schoenlein, J. G. Fujimoto, G. L. Eesley, and T. W. Capehart, Femtosecond dynamics of the $n=2$ image-potential state on Ag(100), Phys. Rev. B 41, 5436 (1990).

[64] G. A. Sawatzky and J. W. Allen, Magnitude and Origin of the Band Gap in NiO, Phys. Rev. Lett. 53, 2339 (1984).

[65] S. Hüfner, J. Osterwalder, T. Riesterer, and F. Hulliger, Photoemission and inverse photoemission spectroscopy of NiO, Solid State Commun. 52, 793 (1984). 
[66] F. Reinert, P. Steinert, S. Hüfner, H. Schmitt, J. Fink, M. Knupfer, P. Sandl, and E. Bertel, Electron and hole doping in NiO, Z. Phys. B 97, 83 (1995).

[67] X. Ren, I. Leonov, G. Keller, M. Kollar, I. Nekrasov, and D. Vollhardt, LDA + DMFT computation of the electronic spectrum of NiO, Phys. Rev. B 74, 195114 (2006).

[68] J. Kuneš, V. I. Anisimov, S. L. Skornyakov, A. V. Lukoyanov, and D. Vollhardt, NiO: Correlated Band Structure of a ChargeTransfer Insulator, Phys. Rev. Lett. 99, 156404 (2007).

[69] M. Däne, M. Lüders, A. Ernst, D. Ködderitzsch, W. M. Temmerman, Z. Szotek, and W. Hergert, Self-interaction correction in multiple scattering theory: Application to transition metal oxides, J. Phys.: Condens. Matter 21, 045604 (2009).

[70] M. W. Cole, Electronic surface states of a dielectric film on a metal substrate, Phys. Rev. B 3, 4418 (1971).

[71] C. B. Harris, N.-H. Ge, R. L. Lingle, J. D. McNeill, and C. M. Wong, Femtosecond dynamics of electrons on surfaces and at interfaces, Annu. Rev. Phys. Chem. 48, 711 (1997).
[72] R. Newman and R. M. Chrenko, Optical properties of nickel oxide, Phys. Rev. 114, 1507 (1959).

[73] P. M. Echenique and J. B. Pendry, Existence and detection of Rydberg states at surfaces, J. Phys. C: Solid State Phys. 11, 2065 (1978).

[74] Ch. Reuß, I. L. Shumay, U. Thomann, M. Kutschera, M. Weinelt, T. Fauster, and U. Höfer, Control of the Dephasing of ImagePotential States by CO Adsorption on $\mathrm{Cu}(100)$, Phys. Rev. Lett. 82, 153 (1999).

[75] M. Weinelt, C. Reuß, M. Kutschera, U. Thomann, I. L. Shumay, T. Fauster, U. Höfer, F. Theilmann, and A. Goldmann, Decay and dephasing of image-potential states due to surface defects and disorder, Appl. Phys. B 68, 377 (1999).

[76] M. Roth, M. Pickel, W. Jinxiong, M. Weinelt, and T. Fauster, Electron Scattering at Steps: Image-Potential States on $\mathrm{Cu}(119)$, Phys. Rev. Lett. 88, 096802 (2002). 